\title{
Decline of seminal parameters in middle-aged males is associated with lower urinary tract symptoms, prostate enlargement and bladder outlet obstruction
}

\author{
Kristo Ausmees, Paul Korrovits, Gennadi Timberg, Margus Punab, Reet Mändar \\ Department of Surgery (KA), Department of Microbiology (PK, RM), Tartu University; Andrology Centre \\ (PK, MP), Tartu University Hospital, Tartu and Department of Surgery, West-Tallinn Central Hospital \\ (GT), Tallinn, Estonia
}

\section{ABSTRACT}

Purpose: We aimed to compare the associations between semen quality, associated reproductive indicators and the main prostate-related parameters in middle-aged men. Materials and Methods: This is a prospective study on 422 middle-aged men who underwent the screening for prostate health. Their reproductive function, semen quality and prostate-related pathologies were investigated.

Results: Significant associations between semen quality and prostate-related parameters could be seen. Total sperm count and sperm density decreased along with the increase of the I-PSS score and total prostate volume. Also, the related lower urinary tract characteristics showed a negative correlation with main semen parameters for all investigated subjects.

No significant differences in age, testicular size, and hormonal parameters were found between the subjects with or without lower urinary tract symptoms and prostate enlargement.

Conclusions: Our study suggests that altered seminal parameters in middle-aged men are associated with LUTS, prostate enlargement and/or bladder outlet obstruction. Although the assessments of prostate and lower urinary tract symptoms may not replace the semen parameters evaluating the male reproductive status, there is a need for further and more detailed investigations about the pathways behind these associations as well as possible related conditions.

\section{ARTICLE INFO}

\section{Key words:}

Reproduction; Semen

Analysis; Middle Aged; Lower

Urinary Tract Symptoms;

Prostatic Hyperplasia

Int Braz J Urol. 2013; 39: 727-40

Submitted for publication:

April 12, 2013

Accepted after revision:

July 24, 2013

\section{INTRODUCTION}

The issue of semen quality of aging men is receiving increasing attention due to trends in prolonged life expectancy in males and the beginning of family planning after the establishment of careers, resulting in higher parental ages. These two issues, in combination with the trend in decreasing semen quality in men $>40$ years, are important factors for increased public awareness of the reproductive status of the aging male $(1,2)$.

Aging is defined by biological and demographic parameters characterized by the impairment of functions of the human body, decreasing environmental responsiveness and, reciprocally, by an increased susceptibility to age-related diseases and mortality (3). In fact, male aging is a multifactorial process that causes changes in di- 
fferent reproductive organs, including the prostate. Although benign prostatic hyperplasia (BPH), a progressive condition characterized by prostate enlargement accompanied by lower urinary tract symptoms (LUTS), is uncommon before age 40, roughly 50\% of men develop BPH-related symptoms by 50 year of age (4). The incidence of BPH increases by $10 \%$ per decade and reaches $80 \%$ by approximately at 80 year of age (5).

Although some recent studies have described the risk of prostatitis and prostate cancer in middle-aged infertile males (6-8), little is known about common associations between reproductive function and prostate pathologies in males $>40$ years. To our best knowledge there are no reports about semen quality and reproductive function in middle-aged males with BPH, bladder outlet obstruction (B00) and/or LUTS.

Therefore, the first aim of this study was to reveal the associations between semen quality, associated reproductive indicators and the main prostate-related parameters in middle-aged men. We also tried to calculate the predictive values of total prostate volume, PSA and I-PSS score to differentiate subjects with and without semen pathologies.

\section{MATERIALS AND METHODS}

\section{Study population}

In the initial phase (between November 2007 and August 2012), 639 men who were submitted to screening for prostate health at the Andrology Centre of Tartu University Hospital were recruited in the study. Exclusion criteria for this study included prior or current problems and/or treatment of infertility or urogenital tumors, chemo- or radiation therapy in the pelvic region, previous varicocelectomy, herniorraphy or vasectomy, history of undescended testicle(s), and/or abnormal findings from a digital rectal examination (DRE). In addition, within the three last months none of the study subjects experienced febrile pelvic pain symptoms and acute urinary retention nor received therapy with antimicrobials, $\alpha 1$-blockers or $5 \alpha$-reductase inhibitors $(9,10)$.

The final group included only the men who were willing to provide semen specimens ( $n=448)$. Among these subjects, 26 males with reported incomplete semen sample (10) were excluded. While the main goal of our study was the screening of prostate health and the additional analyses in middle-aged subjects are mostly disinclined, the assessment of sperm parameters was performed only in a single semen sample; similar methods are described in prior studies of men $>40$ years $(6,11-13)$. However, we re-evaluated the semen quality newly within four weeks after the initial examination.

Finally, the conclusive number of subjects was 422. According to I-PSS score, 380 of them presented with lower urinary tract symptoms and the remaining 42 subjects had no LUTS, respectively. The mean age of participants was $56.1 \pm 6.7(+$ SD) years.

Separation of subjects according to LUTS and total prostate volume

According to lower urinary tract symptoms, the subjects were divided into four groups - without LUTS score (IPSS 0) and with mild (I-PSS score 1-7), moderate (I-PSS score 8-19), and severe (I-PSS score 20-35) symptom scores (9). The separation of subjects according to total prostate volume was made using the cut-off levels of previous large-scale, long-term medical studies $(14,15)$ and the risk for BPH progression (TPV < 30 and $\geq 30 \mathrm{~mL}$, respectively) (16).

\section{Clinical examination}

Physical examinations included assessment of testicular size, genital pathologies, digital rectal examination (DRE), and body mass index (BMI). Participants of the study were examined by one investigator (K.A) who had completed multiple instances of special training on standardization of clinical examinations prior to the described study. The principles of examination have been described previously (13).

\section{Semen analysis}

Semen was obtained by masturbation and ejaculated into a sterile collection tube in a private room near the laboratory. The recommended abstinence period was a minimum of 48 hours but not longer than 7 days. The actual period of ejaculation abstinence was calculated in full days between the current and previous ejaculation as reported by the men.

Routine semen analysis was performed according to WHO guidelines $(10,17)$ to detect semen 
volume, total sperm count, concentration, motility, and morphology. The standpoints of semen analysis have been specified previously (12).

The subjects were studied using the same criteria $(10,17)$ in the Andrology Centre and laboratories. Participants of the study were examined by one technician who had completed multiple instances of special training on laboratory standardization of semen samples prior the study.

\section{Blood samples}

Venous blood was obtained from the cubital vein between 8 a.m. and 11 a.m. after overnight fasting or light morning meal. The samples were centrifuged, serum isolated, and reproductive hormones and prostate specific antigen (PSA) were detected within 2 hours at the United Laboratories of Tartu University Hospital. The levels of follicle stimulating hormone (FSH), luteinizing hormone (LH), testosterone, estradiol (E2), sex-hormone binding globuline (SHBG) and PSA in blood plasma were measured using the Immulite automated chemiluminescence immunoassay analyzer (Immulite Siemens Healthcare Diagnostics Inc, Deerfield, IL, USA) according to the manufacturer's instructions. The intra- and inter-assay coefficients of variation were 4.2 and 8.0\% for FSH, 4.0 and 7.1\% for LH, 6.3 and 9.4\% for testosterone, 7.5 and 13\% for estradiol, 3.4 and $4.1 \%$ for SHBG, and 0.8 and $2.7 \%$ for PSA, respectively.

\section{Questionnaires}

In the initial phase of study, all participants completed the questionnaires, including the Estonian version of National Institutes of Health Chronic Prostatitis Symptom Index (NIH-CPSI) (18) and International Prostate Symptom Score (I-PSS) (9) for lower urinary tract symptoms.

Total prostate volume, post-void residual urine, and urinary flow rates

All men were measured for total prostate volume (TPV) and post-voided residual urine (PVR) by trans-rectal or abdominal ultra-sonography (using Logiq 5 Pro by General Electric, United States) and for urinary flow rates by uroflowmetry (using Urodyn 1000 by Medtronic, United States).

\section{Statistical evaluation}

For statistical analyses, SigmaStat (Systat Software, Chicago, Ill), Excel (Microsoft, Redmond, Wash), and R (R Foundation for Statistical Computing) software programs were used.

In Table-1.1 the median age, different reproductive parameters, and prostate-related characteristics were compared using the Kruskal-Wallis test, and the group or groups that differed from the others were isolated by a multiple comparison procedure (Dunn's method). In Table-1.2, the differences between the groups were compared by the Mann-Whitney test due to nonparametric distribution of variables.

The Spearman product moment correlation was used to determine correlations between age, reproductive, and prostate-related parameters. Areas under receiver operating characteristics (ROC) curves and diagnostic test characteristic $(95 \% \mathrm{Cl})$ for total prostate volume, I-PSS score, and PSA in serum were designed using $\mathrm{R}$ software to estimate semen pathology with cut-off levels of semen volume $<1.5 \mathrm{~mL}$, total sperm count $<39.0$ ( $\times 10^{6}$ per ejaculate), and sperm density $<15$ ( $\times 10^{6}$ per $\left.\mathrm{mL}\right)(10)$.

In the multiple regression analysis, the relations between lower urinary tract symptoms, semen parameters, and related reproductive indicators were analyzed by multiple linear regression models. In statistics, semen parameters were described as dependent variables and other assessments as independent variables.

Statistical significance was assumed at $p$ $<0.05$ for all parameters.

\section{Ethical consideration}

Participation in the study was voluntary. Informed consent was obtained from all study subjects. The study was approved by the Ethics Review Committee on Human Research of the University of Tartu.

\section{RESULTS}

Associations between age, reproductive and prostate-related characteristics

The subject's age, reproductive, and prostate-related characteristics are presented in Table-1. 
All study subjects were grouped according to I-PSS score (Table-1.1) and total prostate volume (Table-1.2).

For described groups, total sperm output and sperm density decreased along with the increase of the I-PSS score and total prostate volume. Also, we found statistical difference between the groups in semen volume, but there was no difference in age, testicular size, body mass index and hormonal parameters.

The correlation coefficients between age, reproductive status, and prostate-related parameters are shown in Table-2. Significant associations between semen quality and prostate-related parameters could be seen. Lower urinary tract characteristics, I-PSS and NIH-CPSI scores, total prostate volume and PSA, showed a negative correlation with main semen parameters for all investigated subjects. Interestingly, maximum urinary flow rate $\left(Q_{\max }\right)$ showed a positive correlation with semen volume, and a negative correlation with time of abstinence before sperm analysis (Table-2).

In addition, total prostate volume was correlated with the PSA level $(r=0.520, p<0.001)$, total I-PSS score $(\mathrm{r}=0.186, \mathrm{p}<0.001)$, and maximum urinary flow rate $(\mathrm{r}=-0.189, \mathrm{p}<0.001)$. There was correlation of I-PSS score, NIH-CPSI score and PSA level with maximum urinary flow rate $(\mathrm{r}=-0.292, \mathrm{p}<0.001, \mathrm{r}=-0.143, \mathrm{p}=0.004$ and $r=-0.292, p<0.001$, respectively), while no correlation of PSA level in serum with I-PSS score and NIH-CPSI score ( $p=0.806$ and $p=0.308$, respectively).

The subject's age had a negative correlation with testicular size $(r=-0.136, p=0.032)$, and sperm parameters, i.e. semen volume $(\mathrm{r}=-0.251$, $\mathrm{p}<0.001)$, and total sperm count $(\mathrm{r}=-0.161, \mathrm{p}=$ $0.001)$.

We did not find any correlation between hormonal and prostate- or sperm-related characteristics in all investigated subjects. Instead, the hormonal parameters were associated with age and BMI. There was a significant positive correlation between age and FSH ( $r=0.220, p<0.001$,), SHBG $(\mathrm{r}=0.170, \mathrm{r}=0.002)$, and E2 levels $(\mathrm{r}=0.162, \mathrm{p}$ $=0.002)$ in serum. BMI showed a negative correlation with testosterone $(\mathrm{r}=-0.201, \mathrm{p}<0.001)$, SHBG ( $r=-0.307, p<0.001)$, sperm motility ( $r$
$=-0.198, p=0.001)$ and lower urinary tract symptoms (Table-2).

The cut-off values of I-PSS, total prostate volume and PSA to detect semen pathologies

The results of the ROC curves for I-PSS, PSA, total prostate volume, and for compounded assessments are presented in Table-3. According to WHO reference ranges (10), speculative cut-off values for I-PSS, PSA in serum, and total prostate volume to detect an abnormal sperm parameters were 14.0 score-points, $1.16 \mathrm{ng} / \mathrm{mL}$, and $41.0 \mathrm{~mL}$, respectively.

The combined ROC curves for I-PSS, PSA, and total prostate volume, constructed using a logistic regression model, showed similar positive and negative predictive values, and AUC-s (0.590.61) for semen pathologies (10).

In addition, a multiple regression analysis was subsequently performed to uncover the significant effects of age and prostate-related parameters on semen quality. The results are summarized in Table-4, indicating similar tendencies revealed by the ROC curve and correlation analyses.

\section{DISCUSSION}

The main finding of our study was that the lower urinary tract parameters are associated with reduced semen volume and sperm parameters in middle-aged men.

According to prolonged lifetimes and delayed family planning, (sub) fertility of an aging couple is an increasing topic for current and further reproductive medicine research. There is a consensus that increased female age is associated with reduced fecundity that becomes clinically relevant in the mid-30s (19). At the same time, there is no consensus about the influence of age on male fertility. Although several studies have shown that sperm quality in men decreases with age $(20,21)$, the trends are not clear. For example, the previous reviews indicated that age may have a negative impact on semen volume, sperm motility and morphology, but data regarding age and sperm concentration are more controversial $(20,21)$. Our study presented the negative effect of male age on semen volume and total sperm count, but not on sperm motility, morphology and concentration. 


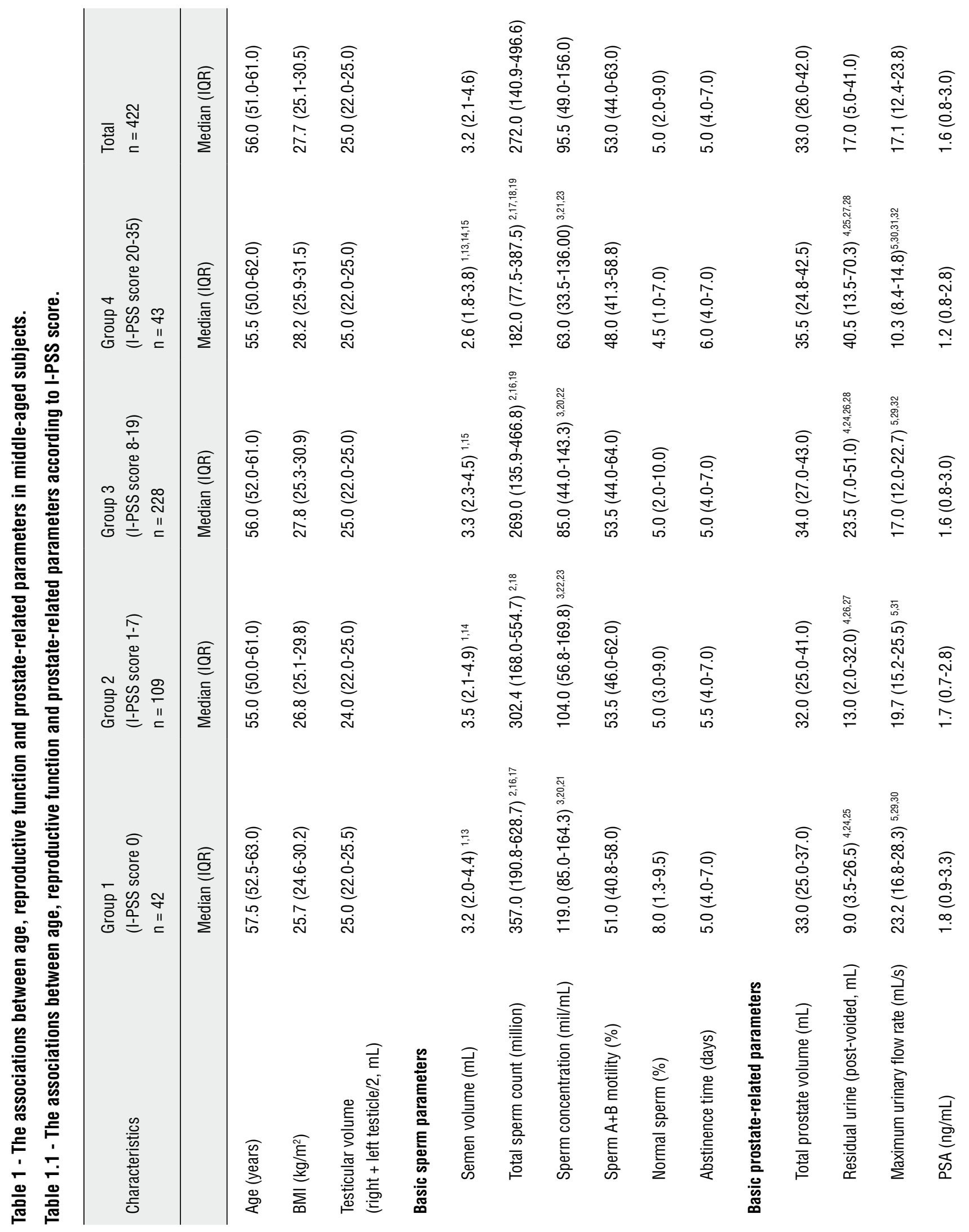




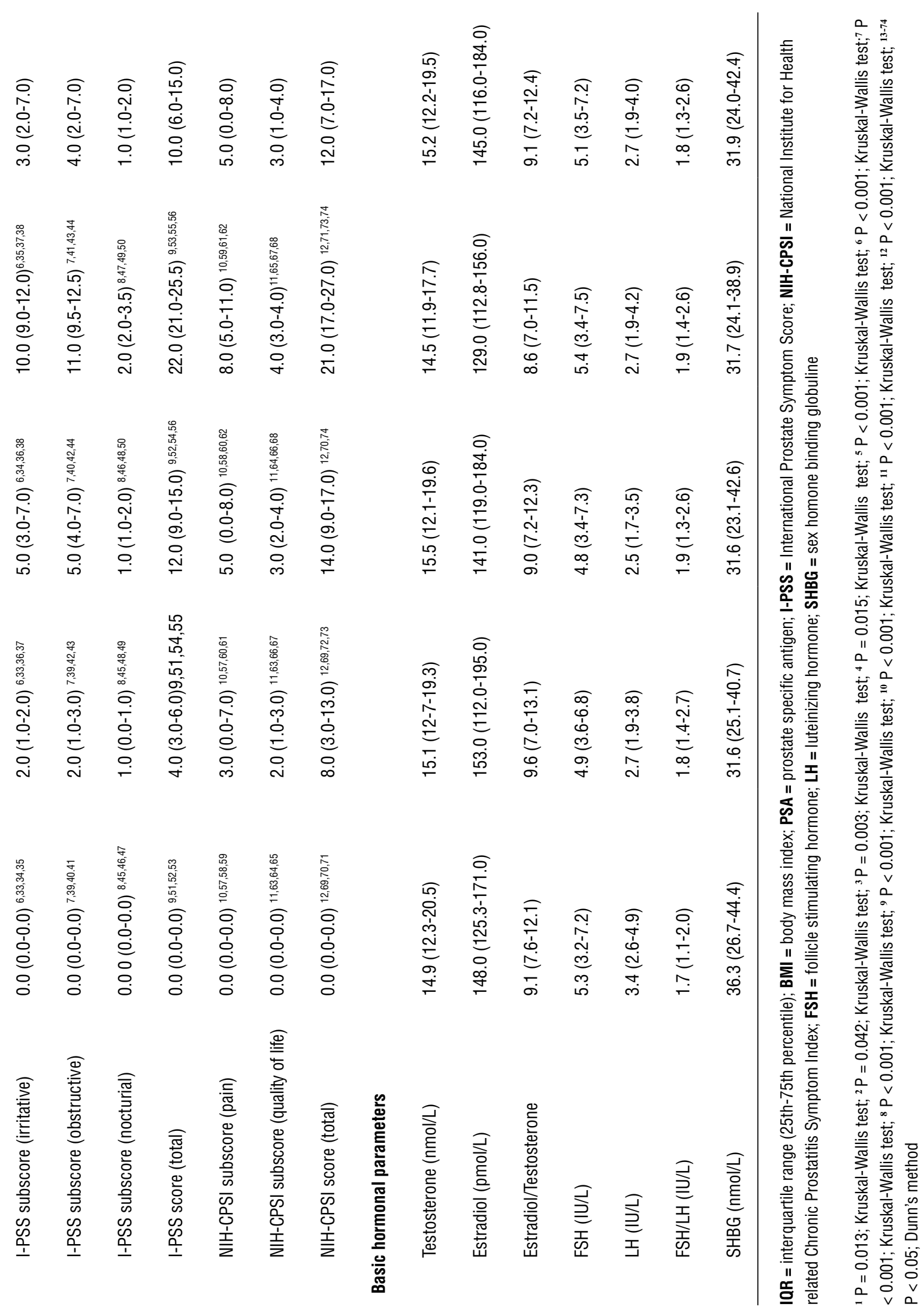




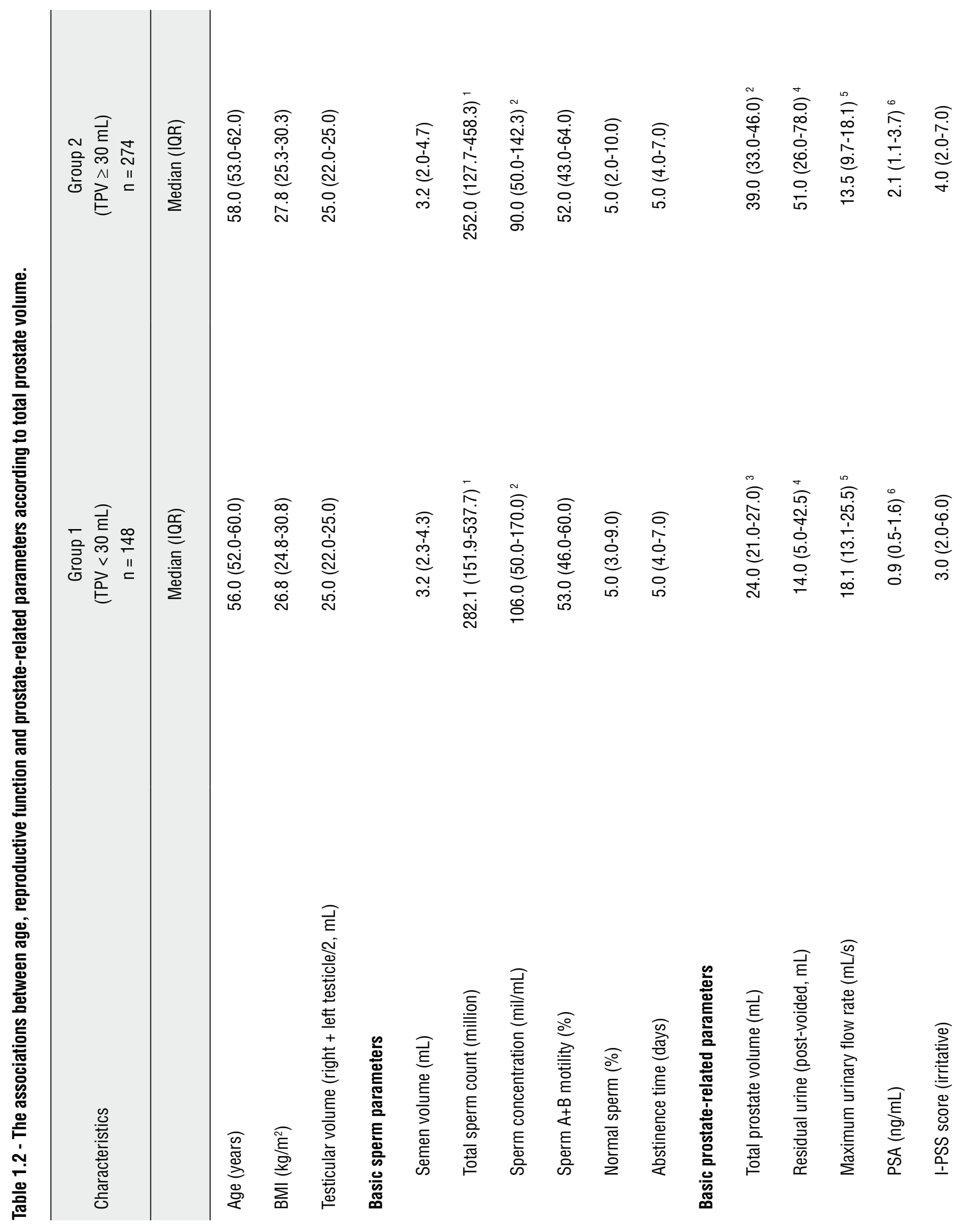




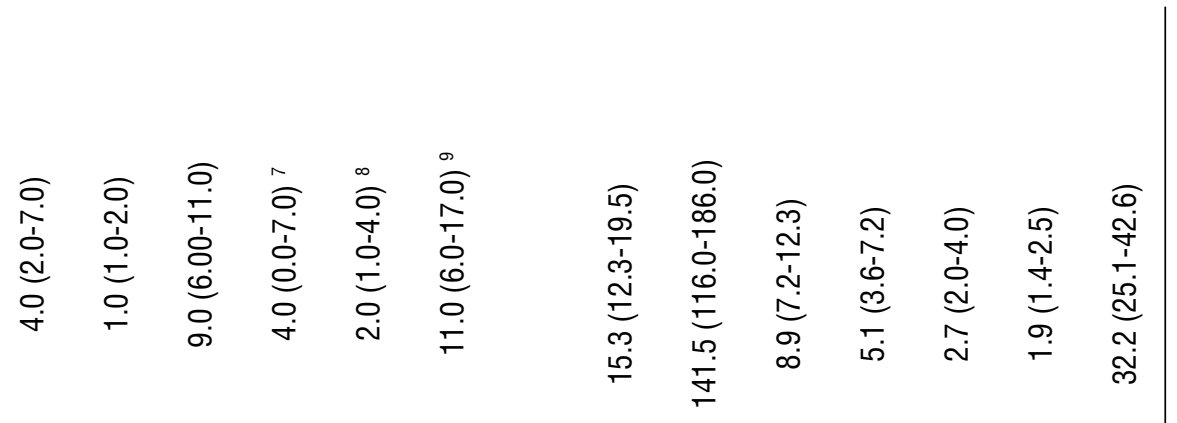

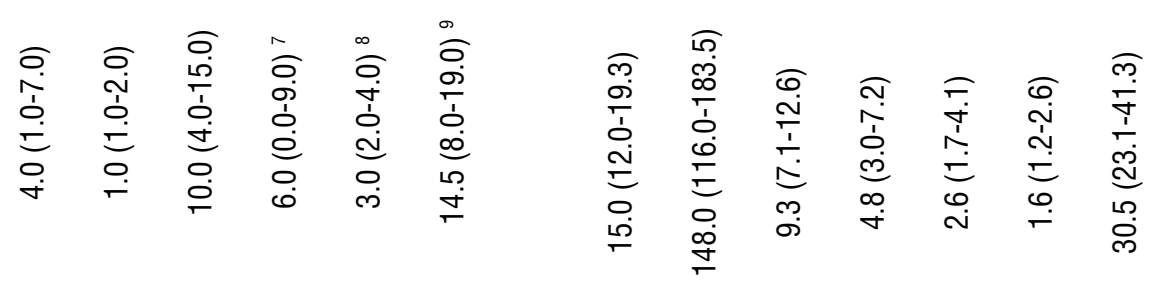

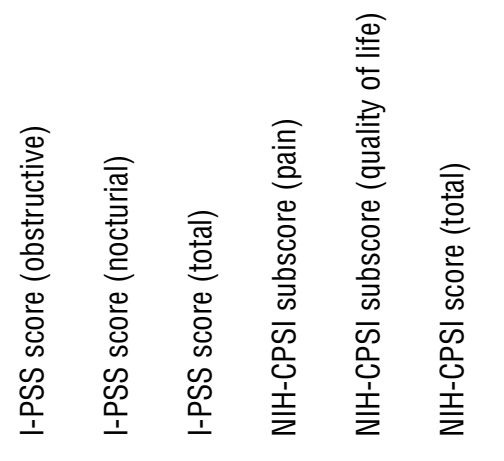

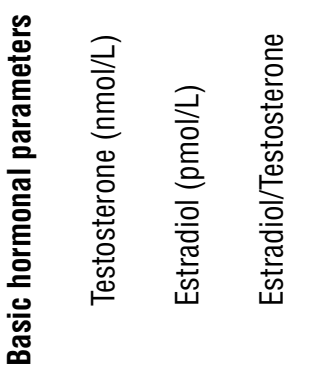

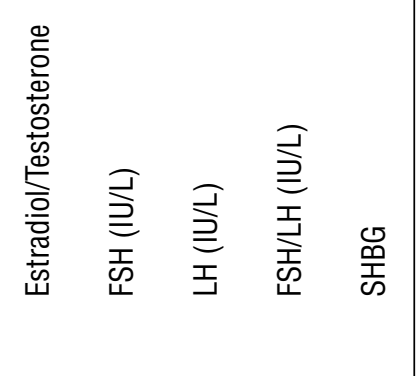

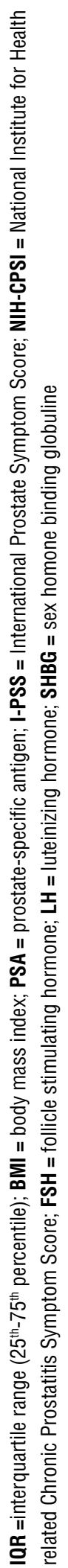

$$
\begin{aligned}
& \text { 离恋 } \\
& \text { 站 今 } \\
& \text { 差 总 } \\
& \text { खे 할 }
\end{aligned}
$$

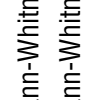

$$
\begin{aligned}
& \text { 产 } \\
& \text { ठै } \\
& \text { il } \\
& \text { 잉 } \\
& \text { 离恋 } \\
& \text { के }
\end{aligned}
$$

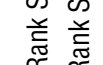

$$
\begin{aligned}
& \text { 항 } \\
& \text { 旁 }
\end{aligned}
$$

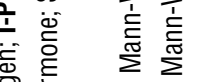

$$
\begin{aligned}
& \text { 총 } \\
& \text { 응 } \\
& \text { "II } \\
& \text { 离苟 } \\
& \text { के } \\
& \text { 亭 亮 }
\end{aligned}
$$

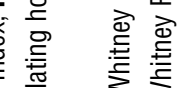

$$
\begin{aligned}
& \text { 亭 空 } \\
& \text { 䎡 } \\
& \text { II } 0 \begin{array}{c}
0 \\
\text { II }
\end{array} \\
& \text { 离芯芯芯 } \\
& \text { ह }
\end{aligned}
$$

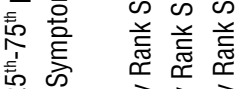

$$
\begin{aligned}
& \text { 品 बे } \\
& \text { 焉 紊紊 }
\end{aligned}
$$

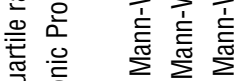

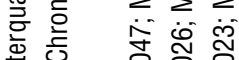




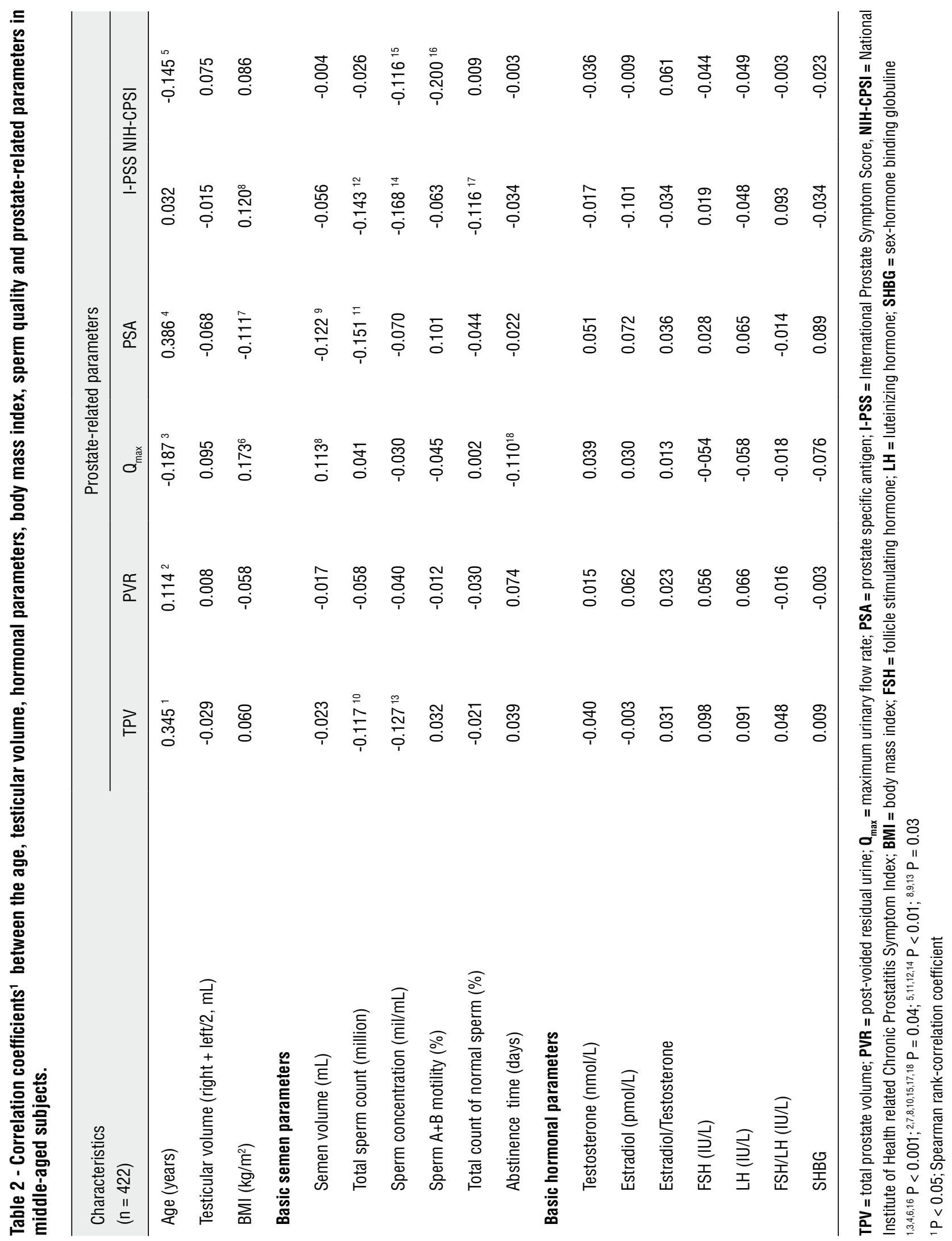


Table 3 - Areas under the ROC curve and diagnostic test characteristics $(95 \% \mathrm{Cl})$ for total prostate volume, I-PSS score, serum PSA level and their combinations as characteristics used to estimate sperm pathology with cut-off levels of semen volume < $1.5 \mathrm{~mL}$, total sperm number < 39.0 (x106 per ejaculate) and sperm density < 15 (x106 per mL) (10).

\begin{tabular}{|c|c|c|c|c|c|c|c|}
\hline $\begin{array}{l}\text { Characteristics } \\
(n=422)\end{array}$ & I-PSS & TVOL & PSA & $\begin{array}{l}\text { I-PSS + } \\
\text { TVOL }\end{array}$ & $\begin{array}{c}\text { I-PSS + } \\
\text { PSA }\end{array}$ & TVOL + PSA & $\begin{array}{l}\text { TVOL + PSA + } \\
\text { I-PSS }\end{array}$ \\
\hline $\begin{array}{l}\text { Sensitivity } \\
\text { (CI 95\%) }\end{array}$ & $\begin{array}{c}39.7 \\
(27.6-52.8)\end{array}$ & $\begin{array}{c}35.5 \\
(23.7-48.7)\end{array}$ & $\begin{array}{c}76.2 \\
(63.8-86.0)\end{array}$ & $\begin{array}{c}69.4 \\
(56.3-80.4)\end{array}$ & $\begin{array}{c}37.1 \\
(25.2-50.3)\end{array}$ & $\begin{array}{c}77.4 \\
(65.0-87.1)\end{array}$ & $\begin{array}{c}56.5 \\
(43.4-69.0)\end{array}$ \\
\hline $\begin{array}{l}\text { Specificity } \\
\text { (CI 95\%) }\end{array}$ & $\begin{array}{c}72.1 \\
(67.176 .8)\end{array}$ & $\begin{array}{c}72.8 \\
(67.8-77.5)\end{array}$ & $\begin{array}{c}42.8 \\
(37.6-48.2)\end{array}$ & $\begin{array}{c}41.2 \\
(36.0-46.6)\end{array}$ & $\begin{array}{c}80.1 \\
(75.5-84.2)\end{array}$ & $\begin{array}{c}41.8 \\
(36.5-47.2)\end{array}$ & $\begin{array}{c}62.6 \\
(57.2-67.7)\end{array}$ \\
\hline $\begin{array}{l}\text { Positive predictive value } \\
\text { (Cl 95\%) }\end{array}$ & $\begin{array}{c}20.5 \\
(13.7-28.7)\end{array}$ & $\begin{array}{c}19.1 \\
(12.4-27.5)\end{array}$ & $\begin{array}{c}19.4 \\
(14.7-24.9)\end{array}$ & $\begin{array}{c}17.6 \\
(13.1-23.0)\end{array}$ & $\begin{array}{c}25.3 \\
(16.7-35.5)\end{array}$ & $\begin{array}{c}19.4 \\
(14.7-24.9)\end{array}$ & $\begin{array}{c}21.5 \\
(15.4-28.6)\end{array}$ \\
\hline $\begin{array}{l}\text { Negative predictive value } \\
(\mathrm{Cl} 95 \%)\end{array}$ & $\begin{array}{c}86.9 \\
(82.4-90.5)\end{array}$ & $\begin{array}{c}86.2 \\
(81.6-89.9)\end{array}$ & $\begin{array}{c}90.9 \\
(85.4-94.8)\end{array}$ & $\begin{array}{c}88.1 \\
(82.1-92.7)\end{array}$ & $\begin{array}{c}87.5 \\
(83.4-91.0)\end{array}$ & $\begin{array}{c}91.1 \\
(85.5-95.0)\end{array}$ & $\begin{array}{c}88.8 \\
(84.1-92.5)\end{array}$ \\
\hline AUC for semen pathology & $\begin{array}{c}54.2 \\
(46.1-62.4)\end{array}$ & $\begin{array}{c}51.5 \\
(43.5-59.6)\end{array}$ & $\begin{array}{c}59.8 \\
(52.4-67.1)\end{array}$ & $\begin{array}{c}54.7 \\
(46.5-62.9)\end{array}$ & $\begin{array}{c}58.4 \\
(50.3-66.5)\end{array}$ & $\begin{array}{c}59.5 \\
(52.1-67.1)\end{array}$ & $\begin{array}{c}58.6 \\
(50.5-66.7)\end{array}$ \\
\hline
\end{tabular}

$\mathbf{C I}=$ confidence intervall; $\mathbf{A U C}=$ area under the curve; $\mathbf{I}-\mathbf{P S S}=$ International Prostate Symptom Score; $\mathbf{T P V}=$ total prostate volume; $\mathbf{P S A}=$ prostate specific antigen

In addition to the impact of age, some previous studies have described anatomical and physiological conditions in reproductive organs in relation to diminished semen quality (20). For example, a decrease of semen volume may be caused by insufficient seminal vesicles, since seminal fluid contributes to most of the ejaculate volume $(20,22)$.

Also, the prostate - an accessory gland of the male reproductive system - is directly related to male reproductive, sexual, and ejaculatory function $(23,24)$. Although previous knowledge is that altered functions in the prostate, such as smooth muscle atrophy and a decrease in protein and water content, may contribute to reduced semen volume and sperm motility (20), there are only a few clinical studies about prostate-related changes on reproductive function and/or sperm quality in middle-aged men $(6-8,11,13)$. While urinary dysfunction is a classic clinical symptom related with prostate pathologies (9), and an association of the latter with reproductive function is presumable, the connections between lower urinary tract symptoms and reproductive quality in middle-aged men have not been previously described.

Our prospective one-center study revealed that prostate enlargement, bladder outlet obstruc- tion and LUTS were related to diminished semen volume and worsened main sperm parameters for all subjects. For example, maximum urinary flow rate, the diagnostic non-invasive assessment describing abnormal voiding and bladder outlet obstruction (B00) (9), showed a significant negative correlation with abstinence time before sperm analysis and a positive correlation with semen volume. Although we have no additional data related to the subject's known lifestyle factors, we may assume that the middle-aged males with longer abstinence time in this study could have rarer sexual intercourses and ejaculation. Therefore, decreased sexual activity could be indirectly related to obstructive symptoms of voiding $\left(Q_{\max }\right)$. In addition, age-related reduction in sexual intercourse and ejaculation frequency may lead to accessory gland pathologies, i.e. prostatitis and bladder outlet obstruction, reduced fluid secretion from the prostate and seminal vesicles, and therefore to decreased semen volume (25). Similar statistically significant associations between BOO and semen parameters have been revealed in multiple regression analysis. However, the assessed prostate-related characteristics showed correlations only with some seminal parameters. Therefore, according to 


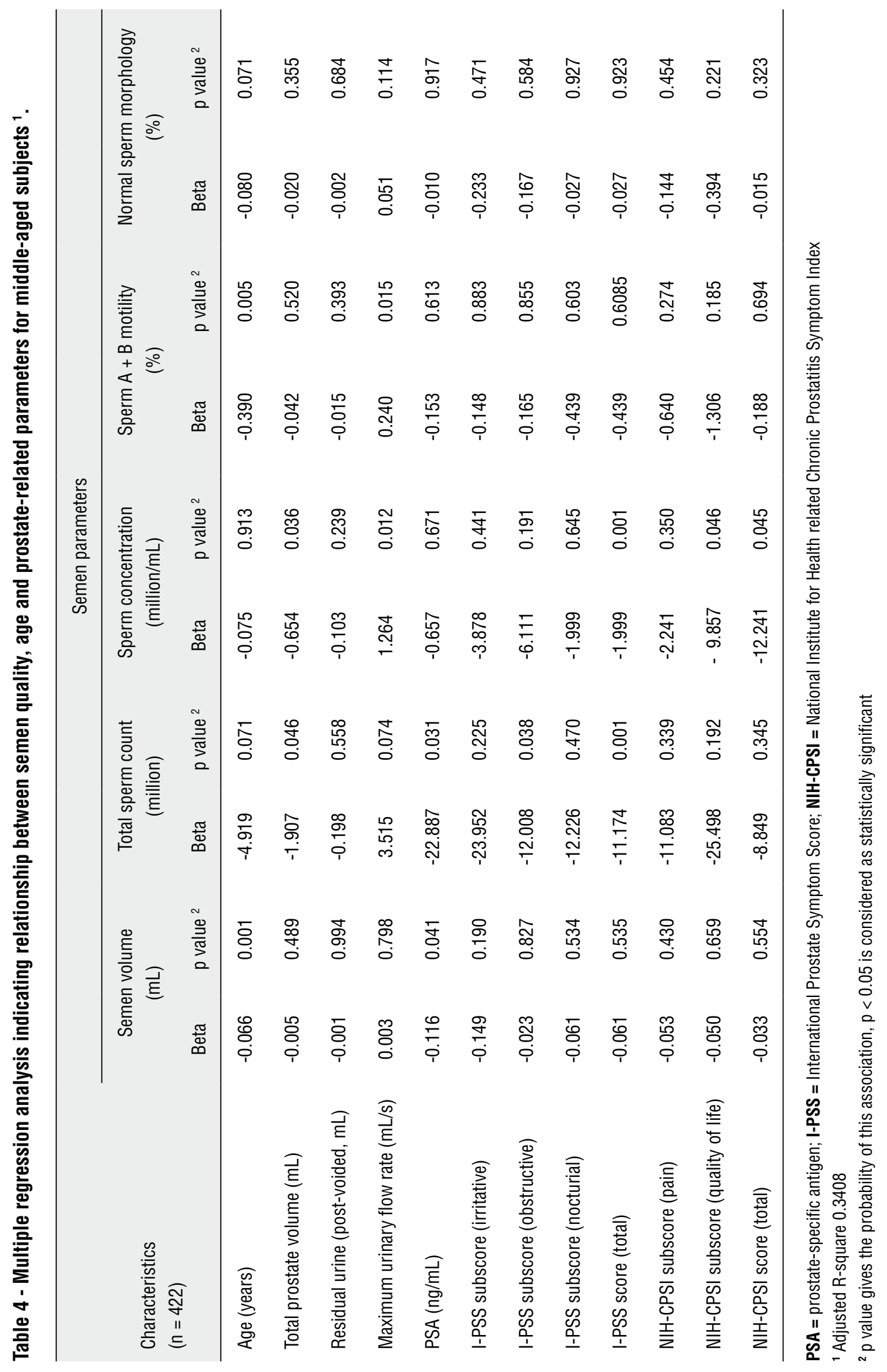


our initial findings, the topic needs more detailed investigations to find out possible pathways as well as possible related conditions.

The essential influences in middle-aged males related both with prostate pathologies and changes in reproductive function are excess weight, increased body mass index $(26,27)$, and dysbalance in sexual hormone levels $(28,29)$. Besides the fact that basic reproductive markers in serum, testosterone and estradiol have been found as important factors for prostate growth (28) and semen quality (29), our study did not reveal any correlation between reproductive hormones and prostate-related parameters for all investigated subjects. Also, there was no correlation between semen parameters and hormonal characteristics.

The present study has a few limitations. First, this study was a one-centre study and therefore related to some organizational problems. The most sophisticated problem was to compose an optimal and operative protocol for the study to assess all parameters related to reproductive quality and lower urinary tract symptoms in males $>45$ years. However, we tried to use well-known and current LUTS parameters and optimal cut-off levels from clinical practice, described in the guidelines of lower urinary tract symptoms (9) and in previous large-scale studies $(14,15)$.

Also, at the beginning of the study we tried to minimize all the possible weaknesses. For example, almost three-quarters of previous studies of reproductive function in middle-aged men did not consider the duration of abstinence before semen analysis $(20,21)$. In our study, the recommended period of abstinence $(10,17)$ was no shorter than 48 hours and no longer than seven days for all participants. At the initial phase of the study, the subjects with reported incomplete semen sample (10) were excluded. During the study, all subjects were studied using the same criteria $(10,17)$, in the same centre and laboratories. In addition, no patient had a digital rectal examination within a 1-week period prior to sampling. Also, none of the study subjects had received antimicrobial, $\alpha 1-$ blockers, or $5 \alpha$-reductase inhibitor therapy within 3 months. While previous reports have shown that reduced semen quality in middle-aged males is mainly related with common aging, alterations in male genital tract and environmental factors $(20,21)$, the data about the prior or current non-reproductive or non-urological diseases and treatment(s) were not added into manuscript.

Second, as has been the case with most previous semen quality studies, the present group included only men who attended a screening in the outpatient clinic and were willing to provide semen specimens, and therefore they do not represent the general population of males aged $>45$ years. While the main goal of our study was the screening of prostate health in middle-aged males and the subjects of that age do not wish mostly to achieve additional analyses, the assessment of sperm parameters was voluntary and performed only in single semen sample. Similar method has been described in prior studies of men $>40$ years [6,11-13]. However, we re-evaluated the semen quality newly within four weeks after initial examination. In fact, almost of all participants showed similar results compared with first semen analysis (data not shown).

\section{CONCLUSIONS}

Our study suggests that altered seminal parameters in middle-aged men are associated with LUTS, prostate enlargement and/or bladder outlet obstruction. Although the assessments of prostate and lower urinary tract symptoms may not replace the semen parameters evaluating the male reproductive status, there is a need for further and more detailed investigations about the pathways behind these associations as well as possible related conditions.

\section{ABBREVIATIONS}

AUC $=$ area under the curve

$\mathrm{BMI}=$ body mass index

BOO = bladder outlet obstruction

$\mathrm{BPH}=$ benign prostatic hyperplasia

DRE $=$ digital rectal examination

E2 = estradiol

$\mathrm{FSH}=$ follicle stimulating hormone

IL-6 = interleukin-6

I-PSS = International Prostate Symptom Score

$\mathrm{LH}=$ luteinizing hormone 
LUTS $=$ lower urinary tract symptoms

NIH-CPSI = National Institutes of Health Chronic

Prostatitis Symptom Index

PSA $=$ prostate specific antigen

PVR $=$ post-voided residual urine

ROC curve $=$ receiver operating characteristic curve

SHBG = sex-hormone binding globuline

$\mathrm{TPV}=$ total prostate volume

\section{ACKNOWLEDGEMENTS}

We are thankful for Heti Pisarev for valuable statistical advice and for Taavo Mägiveer, Mati Kuusemäe, Ülle Poolak and Kadri Poolak for technical assistance. This study was supported by Estonian Ministry of Education and Research (target financing No. SF0180132s08) and Enterprise Estonia (grant No. EU30200).

\section{CONFLICT OF INTEREST}

None declared.

\section{REFERENCES}

1. Lambert SM, Masson P, Fisch H: The male biological clock. World J Urol. 2006; 24: 611-7.

2. Nieschlag E, Behre HM (eds). Andrology: Male reproductive health and dysfunction, 3nd Ed. Springer Verlag, Berlin. 2010; Chapter 14: pp. 239-62.

3. Kirkwood TB: Understanding the odd science of aging. Cell. 2005; 120: 437-47.

4. Parsons JK, Kashefi C: Physical activity, benign prostatic hyperplasia, and lower urinary tract symptoms. Eur Urol. 2008; 53: $1228-35$.

5. Elterman DS, Barkin J, Kaplan SA: ptimizing the management of benign prostatic hyperplasia. Ther Adv Urol. 2012; 4: 77-83.

6. Rolf C, Kenkel S, Nieschlag E: Age-related disease pattern in infertile men: increasing incidence of infections in older patients. Andrologia. 2002; 34: 209-17.

7. Ruhayel $Y$, Giwercman A, Ulmert D, Rylander L, Bjartell A, Manjer J, et al.: Male infertility and prostate cancer risk: a nested case-control study. Cancer Causes Control. 2010; 21: 1635-43.

8. Walsh TJ, Schembri M, Turek PJ, Chan JM, Carroll PR, Smith JF, et al.: Increased risk of high-grade prostate cancer among infertile men. Cancer. 2010; 116: 2140-7.
9. Oelke M, Bachmann A, Dezcazeaud A: Guidelines of the management of Male Lower Urinary Tract Symptoms (LUTS). http://www.uroweb.org/gls/pdf/12_Male_LUTS_LR\%20 May\%209th\%202012.pdf

10. World Health Organization: Laboratory Manual for the Examination and Processing of Human Semen. 5th ed. Geneva, Switzerland. 2011.

11. $\mathrm{Ng} \mathrm{KK}$, Donat R, Chan L, Lalak A, Di Pierro I, Handelsman DJ: Sperm output of older men. Hum Reprod. 2004; 19: 1811-5.

12. Ausmees $K$, Mändar R, Korrovits $P$, Zarkovski M, Timberg G, Punab M: Reproductive function in middle-aged males: healthy men versus male partners of infertile couple. Andrologia. 2012; 20: [Epub ahead of print]

13. Ausmees $\mathrm{K}$, Korrovits $P$, Timberg G, Punab M, Mändar R: Semen quality and associated reproductive indicators in middle-aged males: the role of non-malignant prostate conditionsand genital tract inflammation. World J Urol. 2013; 24: [Epub ahead of print]

14. Roehrborn CG, Marks LS, Fenter T, Freedman S, Tuttle J, Gittleman M, et al.: Efficacy and safety of dutasteride in the four-year treatment of men with benign prostatic hyperplasia. Urology. 2004; 63: 709-15.

15. Roehrborn CG, Barkin J, Siami P, Tubaro A, Wilson TH, Morrill $B B$, et al.: Clinical outcomes after combined therapy with dutasteride plus tamsulosin or either monotherapy in men with benign prostatic hyperplasia (BPH) by baseline characteristics: 4-year results from the randomized, double-blind Combination ofAvodart and Tamsulosin (CombAT) trial. BJU Int. 2011; 107: 946-54.

16. Emberton M, Cornel EB, Bassi PF, Fourcade RO, Gómez JM, Castro R: Benign prostatic hyperplasia as a progressive disease: a guide to the risk factors and options for medical management. Int J Clin Pract. 2008; 62: 1076-86.

17. World Health Organization: Laboratory Manual for Examination of Human Semen and Sperm-Cervical Mucus Interaction. 4th ed. New York, Cambridge University Press. 1999.

18. Korrovits P, Punab M, Mehik A, Mändar R: The Estonian version of the National Institutes of Health chronic prostatitis symptom index. Andrologia. 2006; 38: 106-9.

19. Baird DT, Collins J, Egozcue J, Evers LH, Gianaroli L, Leridon $\mathrm{H}$, et al.: Fertility and ageing. Hum Reprod Update. 2005; 11: 261-76.

20. Sartorius GA, Nieschlag E: Paternal age and reproduction. Hum Reprod Update. 2010; 16: 65-79.

21. Stewart AF, Kim ED: Fertility concerns for the aging male. Urology. 2011; 78: 496-9.

22. Harris ID, Fronczak C, Roth L, Meacham RB: Fertility and the aging male. Rev Urol. 2011; 13: e184-90.

23. McVary K: Lower urinary tract symptoms and sexual dysfunction: epidemiology and pathophysiology. BJU Int. 2006; 97: 23-8; discussion 44-5. 
24. Hellstrom WJ, Giuliano F, Rosen RC: Ejaculatory dysfunction and its association with lower urinary tract symptoms of benign prostatic hyperplasia and BPHtreatment. Urology. 2009; 74: 15-21.

25. Roberts $\mathrm{M}$, Jarvi $\mathrm{K}$ : Steps in the investigation and management of low semen volume in the infertile man. Can Urol Assoc J. 2009; 3: 479-85.

26. Vikram A, Jena G, Ramarao P: Insulin-resistance and benign prostatic hyperplasia: the connection. Eur J Pharmacol. 2010; 641: 75-81.
27. Sallmén M, Sandler DP, Hoppin JA, Blair A, Baird DD: Reduced fertility among overweight and obese men. Epidemiology. 2006; 17: 520-3.

28. Prins GS, Korach KS: The role of estrogens and estrogen receptors in normal prostate growth and disease. Steroids. 2008; 73: 233-44.

29. Jensen TK, Andersson AM, Jørgensen N, Andersen AG, Carlsen $\mathrm{E}$, Petersen $\mathrm{JH}$, et al.: Body mass index in relation to semen quality and reproductive hormones among 1,558 Danish men. Fertil Steril. 2004; 82: 863-70.

Correspondence address: Dr. Reet Mändar

Department of Microbiology University of Tartu Ravila 19, Tartu 50411, Estonia Fax: + 3727 374-172

E-mail: reet.mandar@ut.ee 